\title{
Hydrogenation in supercritical 1,1,1,2 tetrafluoroethane (HFC 134a)
}

\author{
Received 27th May 2005, Accepted 11th August 2005 \\ First published as an Advance Article on the web 2nd September 2005 \\ DOI: $10.1039 / b 507554 h$
}

Andrew P. Abbott, ${ }^{a}$ Wayne Eltringham, ${ }^{a}$ Eric G. Hope ${ }^{* a}$ and Mazin Nicola ${ }^{b}$

Conventional, unmodified, transition metal catalysts, substrates and reagents have sufficient solubility in sc HFC134a for organic synthesis. Reactivities (100\% conversion in $2 \mathrm{~h}$ ) and enantioselectivities ( $c$. 90\%), comparable to those achievable in conventional organic solvents, are obtained in the asymmetric hydrogenation of a series of substrates in this alternative reaction medium using a rhodium(I)/MonoPhos catalyst.

\section{Introduction}

Supercritical fluids (SCFs) have received a huge amount of attention, in both academia and industry, as alternative reaction media for more environmentally friendly chemical processes. $^{1-4}$ The key advantages of SCFs arise from their tuneable polarity, high mass transport rates and environmental compatibility. Most studies have concentrated on $\mathrm{scCO}_{2}$ because of the easily accessible critical constants, cost and low toxicity, exemplified by the recent launch of a $\mathrm{scCO}_{2}$-based commercial hydrogenation process by Thomas Swan. ${ }^{5}$ Whilst the range of reactions evaluated in SCFs is large, the number of examples of asymmetric induction are few ${ }^{1-4}$ and these are virtually all restricted to $\mathrm{scCO}_{2}$. Asymmetric hydrogenation of $\alpha, \beta$-unsaturated carboxylic acids by $\left[\mathrm{Ru}(\mathrm{OAc})_{2}(\mathrm{BINAP})\right]$ in $\mathrm{scCO}_{2}$ was reported to afford low conversions and low enantioselectivities, although both the conversion and ee's could be improved when the ligand was made more $\mathrm{CO}_{2}$-philic by saturating two of the naphthyl rings. ${ }^{6}$ Solubility in $\mathrm{scCO}_{2}$ can also be enhanced by the introduction of perfluoroalkyl substituents, for example in the 6,6'-positions of the BINAP ligand. ${ }^{7,8}$ However, we observed poor reactivity and enantioselectivity in the hydrogenation of dimethyl itaconate in $\mathrm{scCO}_{2}$, which we have ascribed to the apolarity of the solvent. ${ }^{8}$ $\mathrm{We},{ }^{8}$ and others, ${ }^{6,9}$ have observed enhanced conversions and improved enantioselectivities for such ruthenium-catalysed asymmetric hydrogenations in $\mathrm{scCO}_{2}$ by the addition of polar additives, such as methanol. However, here we add a note of caution since, under carefully controlled conditions, in our work it appears that the reaction is essentially finished before the conditions for $\mathrm{scCO}_{2}$ had been reached. ${ }^{8}$ Asymmetric hydrogenation of $\alpha$-enamides with a cationic Rh-DuPHOS catalyst has been made possible by using a highly fluorinated, $\mathrm{CO}_{2}$-philic anion, ${ }^{10}$ and a similar approach has been adopted for the enantioselective hydrogenation of an imine with a cationic iridium catalyst. ${ }^{11}$ We have also shown that the addition of a highly fluorinated anion offers improvements in both reactivity and asymmetric induction in the asymmetric hydrogenation of dimethylitaconate with cationic rhodium complexes containing perfluoroalkylated monodentate

${ }^{a}$ Chemistry Department, University of Leicester, Leicester, UK LE17RH.E-mail:egh1@le.ac.uk; Fax:+441162523789

${ }^{b}$ Advanced Phytonics Ltd, Olway Works, Healey Road, Ossett, West Yorkshire, UK WF5 $8 L T$ phosphinite, phosphite and phosphoramidite ligands, but the results are, at best, modest. ${ }^{12}$

These studies suggest that, for asymmetric hydrogenation at least, the apolarity of $\mathrm{scCO}_{2}$ is a significant issue. However, we have previously shown that some hydrofluorocarbon (HFC) fluids such as difluoromethane and 1,1,1,2 tetrafluoroethane (HFC 134a) are relatively polar solvents, ${ }^{13-15}$ even in the sc state, and this allows them to be used as efficient extraction solvents either on their own or in conjunction with $\mathrm{CO}_{2} \cdot{ }^{16,17}$ Furthermore, these solvents are also readily available and nontoxic. They have easily accessible critical constants (HFC 134a $T_{\mathrm{c}}=374.1 \mathrm{~K} ; p_{\mathrm{c}}=40.6$ bar and $\mathrm{CH}_{2} \mathrm{~F}_{2} T_{\mathrm{c}}=351.1 \mathrm{~K}$; $p_{\mathrm{c}}=57.8$ bar), gaseous dipole moments of about $2 \mathrm{D}^{15}$ and very high densities in the sc state. HFCs have been proposed as useful solvents for synthesis in the sc state and we have recently shown the first examples of the use of $\mathrm{CH}_{2} \mathrm{~F}_{2}$ for the radical polymerisation of methylmethacrylate, ${ }^{18}$ the Friedel Crafts alkylation of anisole ${ }^{19}$ and the esterification of benzoic acid. ${ }^{20}$ Here, we report our results using sc HFC 134a as a convenient, environmentally benign solvent, for enantioselective hydrogenation.

\section{Results and discussion}

The study took two parts. Initially, Wilkinson's catalyst was used for the homogeneous hydrogenation of styrene, as a test reaction, to establish catalytic protocols and the viability of HFC 134a as a solvent for hydrogenation. Subsequently, enantioselective hydrogenation in HFC 134a was evaluated using a rhodium/MonoPhos catalyst generated in situ.

Wilkinson's catalysts have often been used as model systems for the homogeneous hydrogenation of alkenes in liquid solvents ${ }^{21-23}$ and hence it was initially used for the hydrogenation of styrene in sc HFC 134a. Although the solubility of $\left[\mathrm{RhCl}\left(\mathrm{PPh}_{3}\right)_{3}\right]$ in $\mathrm{HFC} 134 \mathrm{a}$ could not be measured quantitatively, a visual inspection using the cell shown in Fig. 1 showed that fully homogeneous solutions of Wilkinson's catalyst in HFC $134 \mathrm{a}$ up to $3 \times 10^{-3} \mathrm{M}$, at $383 \mathrm{~K}$ in the pressure range of 50-280 bar, could be readily prepared. In marked contrast, a similar experiment using $1.3 \times 10^{-3} \mathrm{~mol} \mathrm{dm}^{-3}\left[\mathrm{RhCl}\left(\mathrm{PPh}_{3}\right)_{3}\right]$ in $\mathrm{CO}_{2}$ at $313 \mathrm{~K}$ in the pressure range $50-120$ bar did not show any evidence of catalyst dissolution. This is a significant observation indicating that HFCs can be used as reaction media for homogeneous catalysis without the need for 


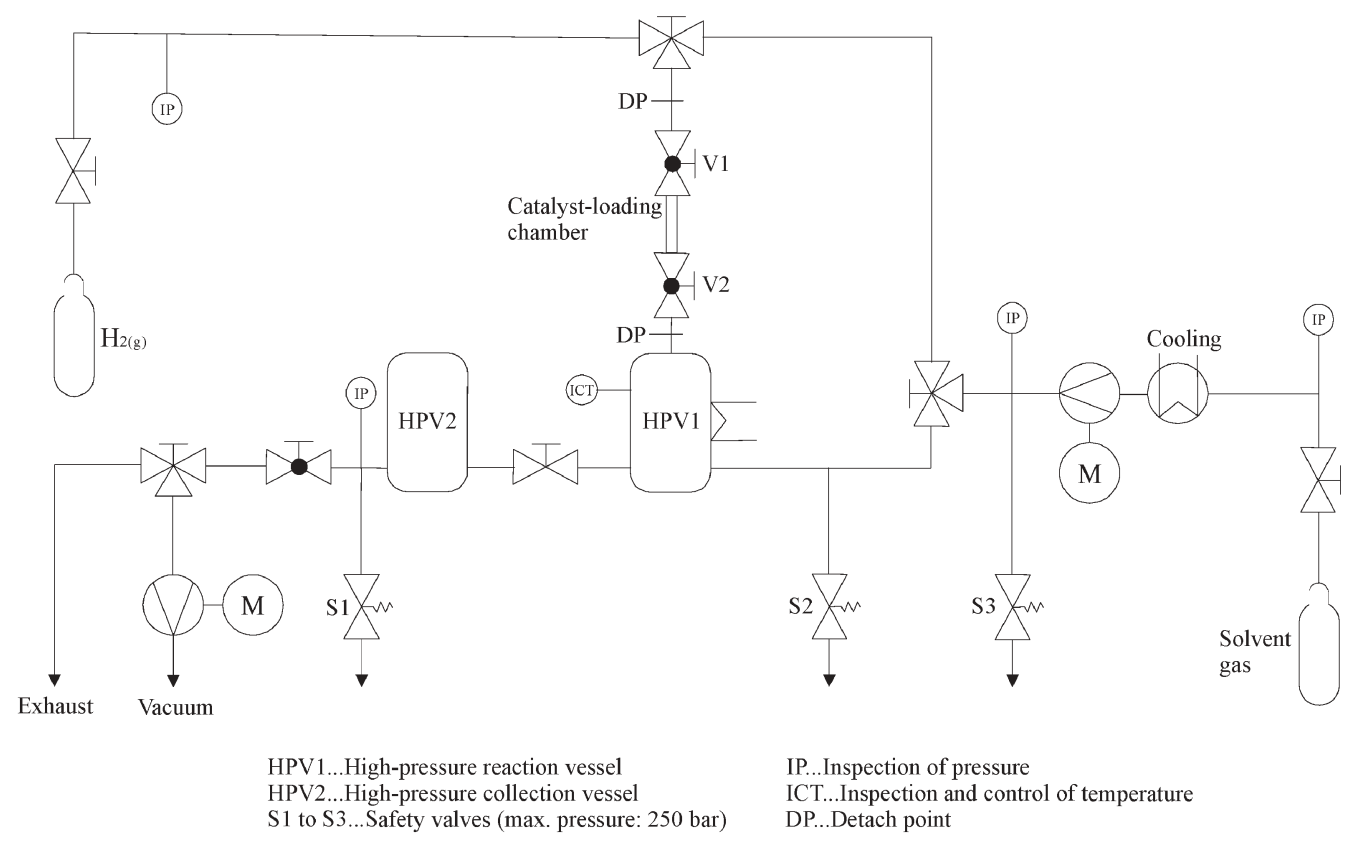

Fig. 1 Schematic diagram of the high-pressure apparatus used in this work.

co-solvents or modification of catalytic species, such as the addition of perfluoroalkyl-ponytails, which is often a requirement for homogeneous catalytic reactions in $\mathrm{scCO}_{2}{ }^{1}$

Firstly, the time dependency of the hydrogenation of styrene $\left(6.56 \times 10^{-3} \mathrm{~mol} \mathrm{\textrm {dm } ^ { - 3 }}\right)$ in sc HFC $134 \mathrm{a}$, using $2.20 \times 10^{-6} \mathrm{~mol} \mathrm{dm}^{-3}$ of $\left[\mathrm{RhCl}\left(\mathrm{PPh}_{3}\right)_{3}\right]$ and $0.57 \mathrm{~mol} \mathrm{dm}^{-3}$ of hydrogen at $383 \mathrm{~K}$ and 100 bar total pressure, was investigated. The results are shown in Fig. 2. It can be seen that the reaction reached maximum conversion after $1.75 \mathrm{~h}$ and, therefore, subsequent hydrogenation reactions were carried out for $2 \mathrm{~h}$ to ensure that the reaction had gone to completion. The time dependency results are generally the average of at least two determinations and a reproducibility of $\pm 3 \%$ was obtained. Analysis of the data in Fig. 2 yields a reaction rate of $167 \pm 5 \mathrm{mmol} \mathrm{dm}{ }^{-1} \mathrm{~h}^{-1}$ which is comparable to that reported for the hydrogenation of styrene in toluene. ${ }^{23}$

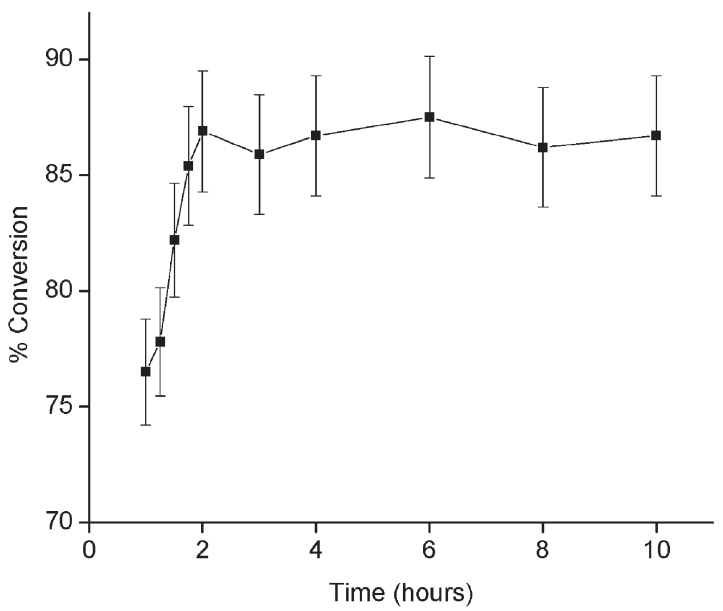

Fig. 2 Time dependency of the hydrogenation of styrene in HFC 134 using $2.20 \times 10^{-6} \mathrm{~mol} \mathrm{dm}^{-3}$ of $\left[\mathrm{RhCl}\left(\mathrm{PPh}_{3}\right)_{3}\right]$ and $0.57 \mathrm{~mol} \mathrm{dm}^{-3}$ of hydrogen at $383 \mathrm{~K}$ and 100 bar total pressure.
The pressure dependency of the hydrogenation of styrene at $383 \mathrm{~K}$ was also investigated and the results are shown in Fig. 3. The results had a reproducibility of $\pm 3 \%$ and the change in dielectric constant with pressure for the pure HFC 134a solvent at $383 \mathrm{~K}$ is also shown. ${ }^{15}$ Reactions were carried out at constant mole fraction in order to observe the effect of pressure on the $\%$ conversion and rule out the possibility of dilution effects as pressure is increased. The mole fractions used are the same as those used in the time dependency study at $383 \mathrm{~K}$ and 100 bar (Fig. 2). It can be seen that the $\%$ conversion increases with increasing pressure and this is attributed to the higher dielectric constant of HFC 134a at higher pressures. It was suggested by Wilkinson et al. ${ }^{21,22}$ that the rate determining step for the hydrogenation of olefins involves the formation of an activated complex, which has a greater dipole moment than the reacting species and that some charge separation is occurring during the formation of the

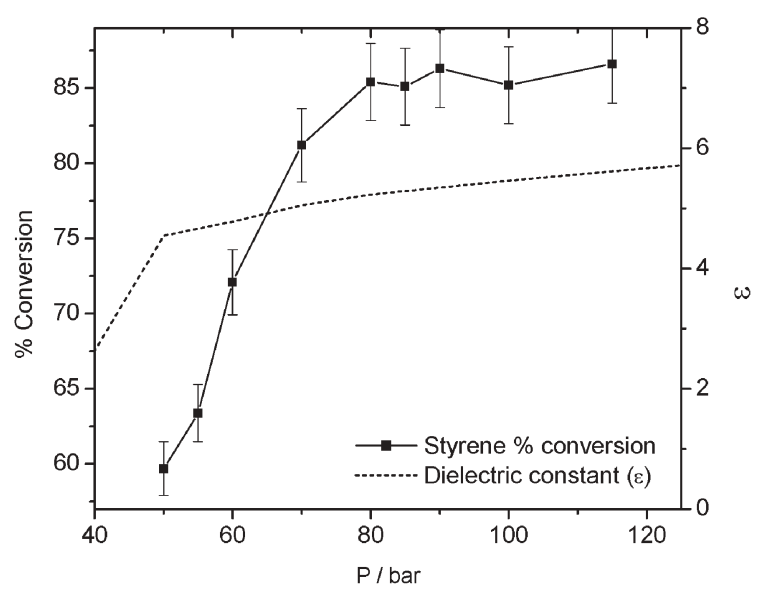

Fig. 3 Pressure dependency of the hydrogenation of styrene using $\left[\mathrm{RhCl}\left(\mathrm{PPh}_{3}\right)_{3}\right]$ in $\mathrm{HFC} 134 \mathrm{a}$ at constant mole fraction and $383 \mathrm{~K}$. 
activated intermediate. The formation of such an activated complex is likely to be more favourable in more polar solvents and is, therefore, more favourable in HFC 134a at higher pressures (higher dielectric constant).

This initial study, the first to use HFC 134a as a reaction medium, demonstrates that both catalytic species and substrates have sufficient solubilities without modification to afford homogeneous hydrogenation conditions, and that reactions rates comparable to those in liquid solvents can be obtained.

The asymmetric hydrogenation studies in sc HFC 134a were performed using $\left[\mathrm{Rh}(\mathrm{COD})_{2} \mathrm{BF}_{4}\right]$ \{bis(1,5-cyclo-octadiene)rhodium(I) tetrafluoroborate $\}$ as the catalyst precursor and the monodentate $(R)$-MonoPhos ligand in a $1: 2$ ratio. MonoPhos, an air- and moisture-stable species, is easily prepared from readily available starting materials and it has been shown that it can facilitate highly enantioselective rhodium-catalysed hydrogenations in conventional solvent systems, ${ }^{24,25}$ whilst $\mathrm{BF}_{4}$ electrolytes have been shown to be highly soluble and extensively dissociated in HFC 134a, ${ }^{26,27}$ making such a rhodium/MonoPhos catalyst ideal for investigating asymmetric hydrogenation in sc HFC 134a. Reactions were carried out using itaconic acid, dimethyl itaconate and (Z)- $\alpha$-acetamido-cinnamic acid (1a, 2a and $\mathbf{3 a}$ respectively in Scheme 1) as model substrates. Prior to the catalytic studies in HFC $134 \mathrm{a}$, we benchmarked the $\operatorname{rhodium} /(R)$-MonoPhos catalytic system and our methodology in the hydrogenation of $\mathbf{1 a}-\mathbf{3 a}$ in conventional organic solvents, where conversions and enantioselectivities very similar to those reported previously were obtained. ${ }^{28}$

In HFC 134a hydrogenations were carried out at $383 \mathrm{~K}$ under a variety of pressures at constant mole fraction, so that dilution effects could be ruled out and the results are shown in Fig. 4 and 5. The results shown for the pressure dependency had a reproducibility of $\pm 4 \%$ whereas those for the enantioselectivity have a larger error of $\pm 8 \%$. It can be seen from Fig. 4 that the $\%$ conversion during the asymmetric hydrogenation reactions shows a similar dependence on pressure as those seen for styrene using $\left[\mathrm{RhCl}\left(\mathrm{PPh}_{3}\right)_{3}\right]$ in Fig. 3 and the same reasoning is offered to explain these trends. For conversions less than $100 \%$ it can be seen that the trend follows the order $\mathbf{1 a}>\mathbf{2} \mathbf{a}>\mathbf{3 a}$ for a given pressure. One possible hypothesis for these observations is that there are two<smiles>C=C(CC(=O)O)C(=O)O</smiles><smiles>C=C(CC(C)=O)CC(=O)OCC(=O)OC(=O)CC(C)C(=O)OC</smiles><smiles>NC(=O)C(=Cc1ccccc1)C(=O)O</smiles>

3a

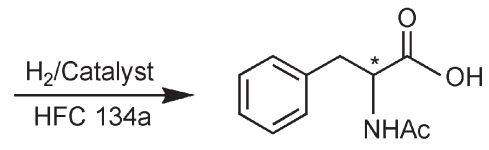

$3 b$

Scheme 1 Reactions studied for asymmetric hydrogenation.

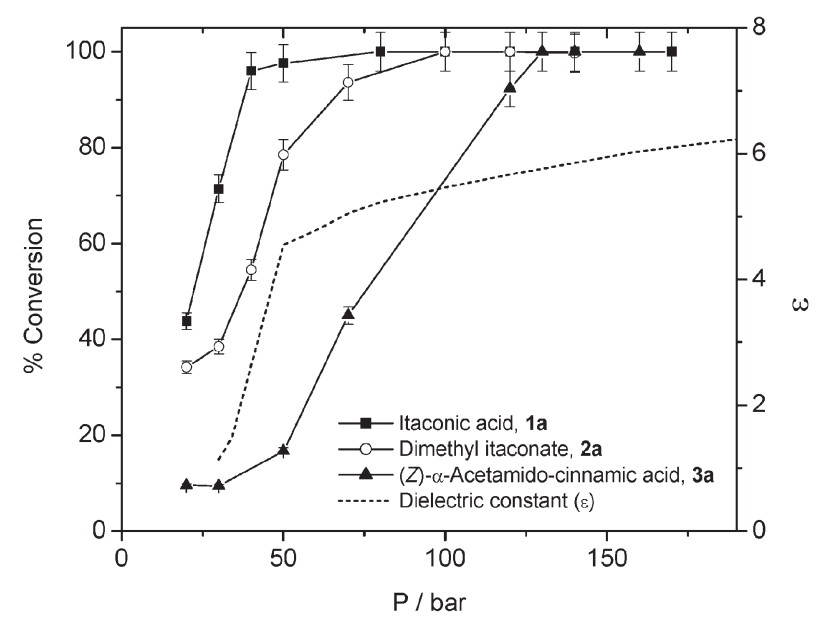

Fig. 4 Pressure dependency of \% conversion for the asymmetric hydrogenation of substrates 1a-3a in HFC 134a at $383 \mathrm{~K}$.

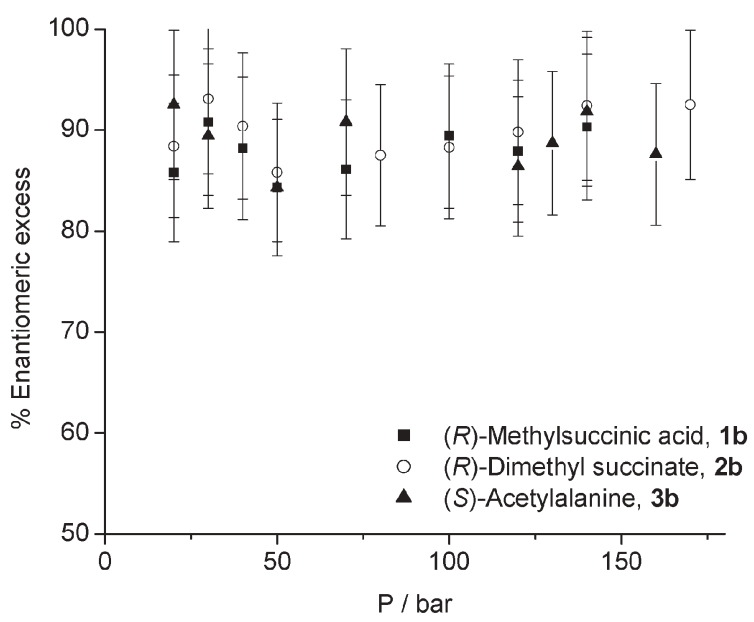

Fig. 5 Pressure dependency of enantiomeric excess for products $\mathbf{1 b}-\mathbf{3 b}$ in HFC $134 \mathrm{a}$ at $383 \mathrm{~K}$.

conflicting influences acting during the reaction; a negative steric effect and the positive effect of increasing dielectric constant. At lower pressures it is suggested that the reaction is under steric control and steric factors are hindering olefinic binding to the rhodium metal centre. The steric hindrance around the position of unsaturation for each substrate follows the trend 1a $<\mathbf{2 a}<\mathbf{3 a}$. At higher pressures the dielectric constant becomes the influencing factor and the $\%$ conversion increases to its maximum value of $100 \%$.

In contrast to our studies on asymmetric hydrogenation in $\mathrm{scCO}_{2},{ }^{8}$ the enantioselectivities for the hydrogenation of $\mathbf{1 a - 3 a}$ in HFC 134a (Fig. 5) are comparable to some of those obtained in liquid solvents, which suggests that HFCs are promising alternative solvents for asymmetric hydrogenation of olefinic substrates. Within experimental error the enantioselectivities at constant mole fraction show no dependency on pressure and, therefore, no dependency on the dielectric constant of HFC 134a. These results are consistent with those of de Vries and co-workers ${ }^{28}$ who carried out solvent screening studies with the rhodium/MonoPhos catalyst using the methyl ester of $(Z)$ - $\alpha$-acetamido-cinnamic acid and found that $\%$ ee 
varied from 70 (methanol) to 95 (DCM) It was concluded that non-protic solvents lead to higher enantioselectivities than protic ones and that the effect of solvent polarity could be ruled out.

It is well known that the concentration of hydrogen can have an effect on the enantioselectivity during asymmetric hydrogenation. ${ }^{29}$ Here, it was found that the $\%$ ee remained roughly constant at $89 \pm 2 \%$, in the hydrogenation of 1 a at $383 \mathrm{~K}$ and 100 bar total, by varying the initial hydrogen pressure from 2 to 20 bar. These results complement those obtained using conventional organic solvents employing rhodium/ MonoPhos $^{24,25,28}$ and rhodium/MonoPhos-based ${ }^{30}$ catalysts that have illustrated that the hydrogen pressure has no effect on enantioselectivity, and suggest that the mechanism of rhodium/MonoPhos catalysis is the same in sc fluids as in liquid solvents.

A study by Poliakoff et $a l^{31}$ showed that the critical parameters of $\mathrm{CO}_{2}$ were reduced during the hydrogenation of propane as the ratios of reagents to products changed. Here, $\alpha$-acetamido-cinnamic acid was used as the substrate in a qualitative set of experiments to investigate the phase behaviour of the HFC 134a hydrogenation system. A windowed vessel was loaded with the same concentration amounts of $3 \mathbf{a}$ and catalyst used for the asymmetric hydrogenation reactions and the system was pressurised with HFC 134a. This process was repeated twice more using the same concentration amount of catalytic material but with a 50/50 substrate/ product mixture and with product alone (emulating the reaction going to completion). It was found from these investigations that, at $383 \mathrm{~K}$, the lowest pressure at which multi-phase behaviour was observed was around 35-38 bar, which is a small reduction on the critical pressure of pure HFC 134a (40.59 bar). Above 38 bar and throughout the pressure dependency studies (Fig. 4) the system was in a single homogeneous phase.

\section{Conclusion}

This work has shown the first example of a reaction in sc HFC 134a. It has been demonstrated that homogeneous catalysts can be used for hydrogenation reactions without the need for co-solvents or fluorous ponytails. The hydrogenation of styrene with Wilkinson's catalyst was used as a model system and it was found that reaction rates comparable to, and in some cases higher than, those in liquid solvents were obtained. The high reaction rates are attributed to the higher diffusivity of gaseous hydrogen in the supercritical media when compared to that in liquid solvents and the percentage conversion is dependent on the solvent dielectric constant, which can be tuned by manipulating the HFC 134a system pressure.

High conversions and enantioselectivities have been obtained for the asymmetric hydrogenation of a range of substrates using a rhodium-MonoPhos catalyst in HFC 134a, which suggests that HFCs are promising alternatives as reaction media for the industrially important asymmetric hydrogenation process. We have recently shown that methylsuccinic acid (1b) and itaconic acid (1a) have significantly different solubilities in HFC 134a and that this has been used to develop an ideal supercritical fluid extraction process, for the separation of reagents and products, using a counter-current separating column. ${ }^{32}$ This, coupled with the high solubility of catalysts and reagents, suggests that HFC $134 \mathrm{a}$ is a useful alternative to $\mathrm{CO}_{2}$ as solvent for reactions in the supercritical state.

\section{Experimental}

$\left(R\right.$-)MonoPhos was prepared by the literature route, ${ }^{33}$ and all other reagents/products were commercial samples and used as received.

The high-pressure cells were constructed from 316 stainless steel and were heated using $240 \mathrm{~V}, 250 \mathrm{~W}$ band heaters supplied by Walton Ltd. The temperature was controlled and maintained $( \pm 0.5 \mathrm{~K})$ using CAL 9900 heater/controllers fitted with an iron/constantan thermocouple, the tip of which was in contact with the solvent close to the centre of the cell. The high-pressure seals between the body of the cells and the cell tops were provided by Teflon ${ }^{\mathbb{R}}$ supported, standard nitrile o-rings. The cells had $3 \mathrm{~cm}$ thick walls, a maximum working pressure of $1 \mathrm{kbar}$ and were rated to $1.5 \mathrm{kbar}$. Each highpressure system was fitted with pressure relief valves set to 300 bar for safety. Pressure was applied using a Thar Technologies P-Series piston pump filled with polytetrafluoroethane (PTFE) composite seals in order to accommodate the use of hydrofluorocarbon (HFC) solvents.

The high-pressure system used for the hydrogenation work is shown schematically in Fig. 1. The catalyst-loading chamber is a $3 \times 0.3 \mathrm{~cm}$ length of stainless steel pipe sealed at each end by a valve. $\mathrm{V}_{1}$ is a two-way needle valve and $\mathrm{V}_{2}$ is a two-way ball valve with a polychlorotrifluoroethylene (PCTFE) seat. The substrate was placed into the reaction vessel and the catalyst was loaded into the catalyst-loading chamber. In the case of air- and moisture-sensitive catalysts the chamber was loaded in a glove box and sealed under nitrogen using $\mathrm{V}_{1}$ and $\mathrm{V}_{2}$. The cell was heated to the desired temperature and evacuated to ensure the reaction vessel was air- and moisturefree. The vessel was charged with the required amount of hydrogen and then pressurised with the appropriate solvent using the P-Series piston pump. The solvent gas was passed through the catalyst-loading chamber, which flushed the catalyst into the reaction vessel, thus starting the reaction. When using HFC solvents, cooling was applied before the gas entered the pump because the pump is more efficient at pumping liquids. The reaction was left for the desired amount of time and the products/unreacted starting materials were collected by depressurisation into the larger volume collection vessel. The products were analysed using NMR (DPX 300), GC-MS (Perkin Elmer) and GC (Perkin Elmer Autosystem XL controlled by Turbochrom software). The enantiomeric excess values for the hydrogenation of dimethyl itaconate and $\alpha$-acetamido-cinnamic acid were determined by GC using a Chiraldex B-DM column. The enantiomeric excess values for the hydrogenation of itaconic acid were determined using polarimetry. Absolute configurations for the itaconic acid and dimethyl itaconate hydrogenation products (methylsuccinic acid and dimethyl succinate) were determined by comparison with commercially available enantiomerically pure 
products and that for acetylalanine, was determined using GC literature data. ${ }^{34}$

The reagent amounts for the constant mole fraction asymmetric hydrogenation studies were $8.25 \times 10^{-5} \mathrm{~mol}$ of substrate and MonoPhos, $4.13 \times 10^{-7} \mathrm{~mol}$ of $\mathrm{Rh}(\mathrm{COD})_{2} \mathrm{BF}_{4}$ and $1.65 \times 10^{-4} \mathrm{~mol}$ of $\mathrm{H}_{2}$ gas for reactions conditions of 100 bar and $383 \mathrm{~K}$. All other reactions were based on these mole fraction values.

In a typical catalytic evaluation, small quantities of reagents were introduced into the cell by dissolving the solid in an appropriate organic solvent and transferring the solution using a Gilson pipette. The air-sensitive catalyst was dissolved in dried and degassed solvents and transferred into the catalystloading chamber in a glove box. The number of moles of hydrogen was converted to pressure values using data from the NIST Chemistry WebBook. ${ }^{35}$

\section{Acknowledgements}

The authors would like to thank Advanced Phytonics Ltd and EPSRC (GR/R 05802) for funding this work.

\section{References}

1 P. G. Jessop, T. Ikariya and R. Noyori, Chem. Rev., 1999, 99, 475.

2 T. Kariy and Y. Kayaki, Catal. Surv. Jpn., 2000, 4, 39.

3 W. Leitner, Acc. Chem. Res., 2002, 35, 746.

4 E. J. Beckman, J. Supercrit. Fluids, 2004, 28, 121.

5 S. K. Ritter, Chem. Eng. News, 2001, 79, 27.

6 J. Xiao, S. C. A. Nefkens, P. G. Jessop, T. Ikariya and R. Noyori, Tetrahedron Lett., 1996, 37, 2813.

7 D. J. Birdsall, E. G. Hope, A. M. Stuart, W. Chen, Y. Hu and J. Xiao, Tetrahedron Lett., 2001, 42, 8551.

8 Y. Hu, D. J. Birdsall, A. M. Stuart, E. G. Hope and J. Xiao, J. Mol. Catal. A, 2004, 219, 57.

9 X. Dong and C. Erkey, J. Mol. Catal. A, 2004, 211, 73.

10 M. J. Burk, S. Feng, M. F. Gross and W. Tumas, J. Am. Chem. Soc., 1995, 117, 8277.

11 S. Kainz, A. Brinkmann, W. Leitner and A. Pfaltz, J. Am. Chem. Soc., 1999, 121, 1999.
12 D. J. Adams, W. Chen, E. G. Hope, A. J. West, J. Xiao and A. M. Stuart, Green Chem., 2003, 5, 118.

13 A. P. Abbott and C. A. Eardley, J. Phys. Chem B, 1998, 102, 8574.

14 A. P. Abbott and C. A. Eardley, J. Phys. Chem, B, 1999, 103, 2504.

15 A. P. Abbott, C. A. Eardley and R. Tooth, J. Chem. Eng. Data, 1999, 44, 112.

16 A. P. Abbott, C. A. Eardley and J. E. Scheirer, J. Phys. Chem., B, $1999, \mathbf{1 0 3}, 8790$

17 A. P. Abbott, C. A. Eardley and J. E. Scheirer, Green Chem., 2000, 2, 63.

18 A. P. Abbott, P. W. Dyer, E. G. Hope, S. Lange and S. Vukusic, Green Chem., 2004, 6, 81.

19 A. P. Abbott, N. E. Durling and E. G. Hope, Chem. Phys. Chem., $2005,6,466$

20 A. P. Abbott, N. E. Durling and E. G. Hope, J. Phys. Chem. B, $2004, \mathbf{1 0 8}, 4922$.

21 S. Montelatici, A. van der Ent, J. A. Osborn and G. Wilkinson, J. Chem. Soc. A, 1968, 1054.

22 G. Wilkinson, R. D. Gillard and J. A. McCleverty, Comprehensive Coordination Chemistry, Pergamon Press, Oxford, 1987.

23 E. G. Hope, D. W. Kemmit, D. R. Paige and A. M. Stuart, J. Fluorine Chem., 1999, 99, 197.

24 X. Jia, R. Guo, X. Li, X. Yao and A. S. C. Chan, Tetrahedron Lett., 2002, 43, 5541.

25 A.-G. Hu, Y. Fu, J.-H. Xie, H. Zhou, L.-X. Wang and Q.-L. Zhou, Angew. Chem. Int. Ed. Engl., 2002, 41, 2348.

26 A. P. Abbott and C. A. Eardley, J. Phys. Chem. B, 2000, 104, 9351.

27 A. P. Abbott, C. A. Eardley, J. C. Harper and E. G. Hope, J. Electroanal. Chem., 1998, 457, 1.

28 M. van den Berg, A. J. Minnaard, E. P. Schudde, J. van Esch, A. H. M. de Vries, J. G. de Vries and B. L. Feringa, J. Am. Chem. Soc., 2000, 122, 11539

29 R. Noyori, Asymmetric Catalysis in Organic Synthesis, John Wiley and Sons, New York, 1994.

30 Q. Zeng, H. Liu, A. Mi, Y. Jiang, X. Li, M. C. K. Choi and A. S. C. Chan, Tetrahedron, 2002, 58, 8799.

31 J. Ke, W. George, M. Poliakoff, B. Han and H. Yan, J. Phys. Chem. B, 2002, 106, 4496.

32 A. P. Abbott, W. Eltringham, E. G. Hope and M. Nicola, Green Chem., 2005, 7, 210.

33 R. Hulst, N. Koen de Vries and B. L. Feringa, Tetrahedron: Asymmetry, 1994, 5, 699.

$34 \alpha$-Acetamido-cinnamic acid is a test substrate used by Chiraldex and the order of elution and separation conditions for the Chiraldex B-DM column are given in the Chiraldex handbook Absolute configuration was determined using the handbook data.

$35 \mathrm{http}: / /$ webbook.nist.gov/chemistry/. 